\title{
ANÁLISE DE SITUAÇÕES DE PROPORÇÃO SIMPLES EM PROVAS DE MATEMÁTICA DO ENEM
}

\section{ANALYSIS OF SIMPLE PROPORTION SITUATIONS IN ENEM MATHEMATICAL TESTS}

\author{
José Diego Martins da Silva ${ }^{1}$; Ernani Martins dos Santos ${ }^{2}$
}

\begin{abstract}
RESUMO
Analisar e compreender as diferentes formas de resolução de um problema matemático, juntamente com o entendimento do conceito envolvido na situação proposta, é de muita relevância para o desenvolvimento dos estudantes. Nesse contexto, Gérard Vergnaud, psicólogo e matemático francês, dá uma grande importância à resolução de situações-problema a partir de campos conceituais (ferramenta teórica que explica como os estudantes constroem conceitos matemáticos e, consequentemente, aprendem matemática no contexto escolar). Isto porque, segundo sua teoria o conhecimento emerge a partir da resolução de problemas. Este trabalho buscou analisar a natureza dos problemas matemáticos, inseridos no Campo Conceitual das Estruturas Multiplicativas, presentes nas provas de matemática e suas tecnologias do ENEM, tendo como foco principal os problemas de relação quaternária do eixo proporção simples. A pesquisa foi desenvolvida dentro de uma abordagem qualitativa, de caráter exploratórioexplicativo, buscando a natureza dos problemas de estruturas multiplicativas, em específico as situações por eixo, incluindo-se as classes, obtidas da análise de cada uma das provas objeto de estudo. Um conjunto de tabelas-síntese serviu de base para categorização e análise das questões identificadas nas provas analisadas. Como principais resultados, temos nestas provas um número de itens, que aborda conceitos multiplicativos, considerado muito satisfatório e que as situações de relação quaternária abordaram especificamente a Proporção Simples enquanto eixo, com pouca sofisticação. Sobre as classes, há uma maior abordagem de situações de um para muitos, sendo requerido em muitos dos itens o emprego do raciocínio proporcional com a presença de mais de uma classe numa mesma situação. Nesta direção, inferimos que a metodologia da resolução de problemas ainda precisa ser mais explorada no processo de ensino e aprendizagem, sob a ótica dos conceitos envolvidos nas situações abordadas, independente das habilidades e competência requeridas na resolução dos problemas.
\end{abstract}

Palavras-chave: ENEM; Campo Conceitual Multiplicativo; Proporção Simples.

\footnotetext{
${ }^{1}$ Graduando em Matemática na Universidade de Pernambuco (UPE). Bolsista de Iniciação Científica do PIBC/CNPq/UPE. Nazaré da Mata, Pernambuco, Brasil. Rua José Luiz Martins, Travessa C, 42. Maria das Neves, Feira Nova, Pernambuco, Brasil. CEP: 55715-000. E-mail: dm80642@gmail.com

ORCID iD: https://orcid.org/0000-0002-2058-3560

${ }^{2}$ Doutor em Psicologia Cognitiva, Universidade Federal de Pernambuco (UFPE). Professor Adjunto e Próreitor de Graduação, atuando no Programa de Pós-graduação em Formação de Professores e Práticas Interdisciplinares e na Graduação em Matemática da Universidade de Pernambuco (UPE), Nazaré da Mata, Pernambuco, Brasil. Rua Marquês de Alegrete, 137, apto 603, Pina, Recife, Pernambuco, Brasil, CEP: 51110-380. E-mail: ernani.santos@upe.br

(iD) ORCID iD: https://orcid.org/0000-0002-3824-986X
} 


\begin{abstract}
Analyzing and understanding the different ways of solving a mathematical problem, along with the understanding of the concept involved in the proposed situation, is of great relevance to the development of students. In this context, Gérard Vergnaud, French psychologist and mathematician, gives great importance to the resolution of problem situations from conceptual fields (a theoretical tool that explains how students build mathematical concepts and, consequently, learn mathematics in the school context). This is because, according to his theory, knowledge emerges from problem solving. This paper sought to analyze the nature of mathematical problems, inserted in the Conceptual Field of Multiplicative Structures, present in the tests of mathematics and its technologies of ENEM, focusing mainly on problems of quaternary relations of the simple proportion axis. The research was developed within a qualitative, exploratory-explanatory approach, seeking the nature of the multiplicative structures problems, specifically the situations per axis, including classes, obtained from the analysis of each of the tests under study. A set of summary tables served as a basis for categorizing and analyzing the questions identified in the analyzed tests. As main results, we have in these tests a very satisfactory number of items that deal with multiplicative concepts and that the quaternary relation situations specifically dealt with Simple Proportion as an axis, with little sophistication. Regarding classes, there is a greater approach to one-to-many situations, and many of the items require the use of proportional reasoning with the presence of more than one class in the same situation. In this sense, we infer that the problem solving methodology still needs to be further explored in the teaching and learning process, from the point of view of the concepts involved in the addressed situations, regardless of the skills and competence required in problem solving.
\end{abstract}

Keywords: ENEM; Multiplicative Conceptual Field; Simple Proportion. 


\section{Introdução}

O Exame Nacional do Ensino Médio (ENEM) teve sua primeira edição em 1998, com o intuito de avaliar a qualidade do Ensino Médio ofertado em nosso país e nos dias atuais serve como forma de ingresso no Ensino Superior de várias universidades brasileiras (públicas e privadas). A prova de matemática e suas tecnologias, nesse exame, é composta por 45 (quarenta e cinco) questões de múltipla escolha, que averiguam o domínio de sete competências e trinta habilidades que abarcam o saber matemático escolar, abordado na Educação Básica, perpassando por cinco blocos temáticos (conhecimento numéricos, geométricos, de estatística e probabilidade, algébricos e algébricos/geométricos) quanto aos objetos de conhecimento que permeiam a matriz de referência.

Analisar e compreender as diferentes formas de resolução de um problema matemático $^{3}$, juntamente com o entendimento do conceito envolvido na situação proposta, é de muita relevância para o desenvolvimento dos estudantes, uma vez que isso permite o aprendizado através da reflexão.

$\mathrm{Na}$ direção de desenvolver habilidades e competências, compreendendo o estudante como um ser pensante e construtor do seu próprio conhecimento, a resolução de problemas matemáticos surge como um recurso propiciador da aprendizagem, sendo reconhecida por diversos estudiosos (POLYA, 1995; D’AMBRÓSIO, 1996; DEMO, 2000; VERGNAUD, 2003; DANTE, 2009) como uma perspectiva metodológica, que constrói estratégias que norteiam o trabalho do professor de forma a garantir a apropriação do conhecimento matemático pelos estudantes e, desta forma, é um ótimo recurso didático para apreender conceitos e procedimentos.

A resolução de problemas tem um valor indiscutível, na ótica da Educação e da Psicologia, como claramente é descrito nos pressupostos da Teoria dos Campos Conceituais (VERGNAUD, 1983; 1988; 1991; 1994; 1996; 2009). Sob esta ótica, Spinillo et. al. (2017, p. 930) descrevem os problemas matemáticos como "situações que

\footnotetext{
${ }^{3}$ Nas provas do ENEM, qualquer situação a ser "resolvida" por uma sequência ações a ser executada, para atingir um objetivo (identificar uma única resposta como correta), onde a situação é o estado inicial e o objeto é o estado final desejado é entendida como problema. Assim, nos itens dessa avaliação, problemas e situações-problema são tomados como sinônimos, bem como neste artigo, tendo em vista que Vergnaud (1996) denomina situação como tarefa ou conjunto de tarefas, subordinada ao conceito matemático envolvido, em que são requeridos esquemas de ações para a obtenção de um objetivo.
} 
tornam os conceitos significativos para o indivíduo, mobilizando um conjunto de operações e representações para sua resolução".

A Teoria dos Campos Conceituais dá uma grande importância à resolução de situações-problema, isso porque, o conhecimento emerge a partir da resolução de problemas, iniciando-se com uma validade restrita e são esses problemas que dão sentido aos conceitos presentes na estrutura cognitiva do indivíduo (VERGNAUD, 1983).

A partir dos pressupostos até aqui descritos, este trabalho buscou analisar a natureza dos problemas matemáticos, inseridos no Campo Conceitual das Estruturas Multiplicativas, presentes nas provas de matemática e suas tecnologias do ENEM, especificamente no triênio 2017-2019, período que compreende um ciclo completo do Ensino Médio (três ano) e que corresponde as últimas edições do exame anteriores ao estudo, tendo como foco principal a análise dos problemas de relação quaternária do eixo proporção simples, a partir da identificação das classes, grandezas e procedimentos.

\section{A Teoria dos Campos Conceituais}

Um Campo Conceitual é entendido como "um conjunto de situações cujo domínio requer uma variedade de conceitos, procedimentos e representações simbólicas firmemente unidas uns aos outros" (VERGNANUD, 1983, p.12).

Esta teoria é considerada construtivista e apresenta um excelente amparo didático, isso porque propõe uma ferramenta teórica que explica como os estudantes constroem conceitos matemáticos e, consequentemente, aprendem matemática no contexto escolar, tendo como base a ideia de que o conhecimento está organizado em campos conceituais que são compostos por várias situações, conceitos, relações, estruturas, conteúdos e operações de pensamentos. Nesta direção, vários pesquisadores brasileiros recentemente têm se dedicado ao estudo desta teoria abordando várias vertentes dos campos conceituais (GITIRANA, et al, 2014; MAGINA; SANTOS; MERLINE, 2016; SANTANA et al, 2016; LAUTERT; SANTOS, 2017; SPINILLO, et al, 2017; BARRETO; RÊGO; 2020).

Estudos sobre os campos conceituais têm evidenciado que a situação comporta uma diversificada rede de conceitos, que vão compor a sua estrutura e, desta forma, um mesmo conceito pode ser identificado em situações distintas. Desta forma, o indivíduo só constrói conceitos no caso dele ser capaz de identificá-lo e, a partir de então, conseguir 
utilizá-lo para a resolução das possíveis situações-problema em que este conceito estará presente.

Salientamos que os campos conceituais não são exclusivos da Matemática como indica Vergnaud (1988), eles permeiam a Física, a Química, a Biologia, etc. Porém, foi na Matemática que Vergnaud dedicou seus estudos, evidenciando os campos conceituais que estruturam a aritmética elementar. Para esse teórico, o campo conceitual da aritmética, apresenta duas estruturas que envolvem um conjunto de situações que requer o uso de determinadas operações, a saber: as Estruturas Aditivas e as Estruturas Multiplicativas, estando o conceito de Proporção Simples, foco do presente estudo, inserido neste último campo conceitual.

\section{O Campo Conceitual Multiplicativo e as Situações de Proporção Simples}

O Campo Conceitual Multiplicativo é definido por Vergnaud (1996, p.19) como um conjunto de situações "que podem ser resolvidas com o uso de uma ou de várias multiplicações ou divisões e os conceitos e teoremas que permitem analisar e resolvê-las, como por exemplo: proporção simples, proporção múltipla, fração, múltiplo, divisor, entre outros".

Diferentemente do raciocínio aditivo, que sinteticamente se resume nas ideias de unir ou separar objetos e conjuntos, em que o todo é igual a soma das partes, o raciocínio multiplicativo assume formas diferentes em três tipos principais de situações: situações de correspondência, situações que envolvem relações entre variáveis e situações de distribuição que, segundo Vergnaud (1983; 1988; 2009), irão requerer um longo período de tempo para a sua apropriação conceitual.

Para melhor compreender a amplitude e as estruturas conceituais que se referem ao Campo Conceitual Multiplicativo, Magina, Santos e Merlini (2016) realizaram uma releitura dos estudos realizados por Vergnaud (1983, 1996, 2009) e elaboraram um esquema (figura 01), com o intuito de resumir as ideias fundamentais das estruturas multiplicativas. Dentre os conceitos trabalhados nesse campo, nosso foco está nas relações quaternárias, mais especificamente no eixo de Proporção Simples. 
Figura 1- Esquema do Campo Conceitual das Estruturas Multiplicativas

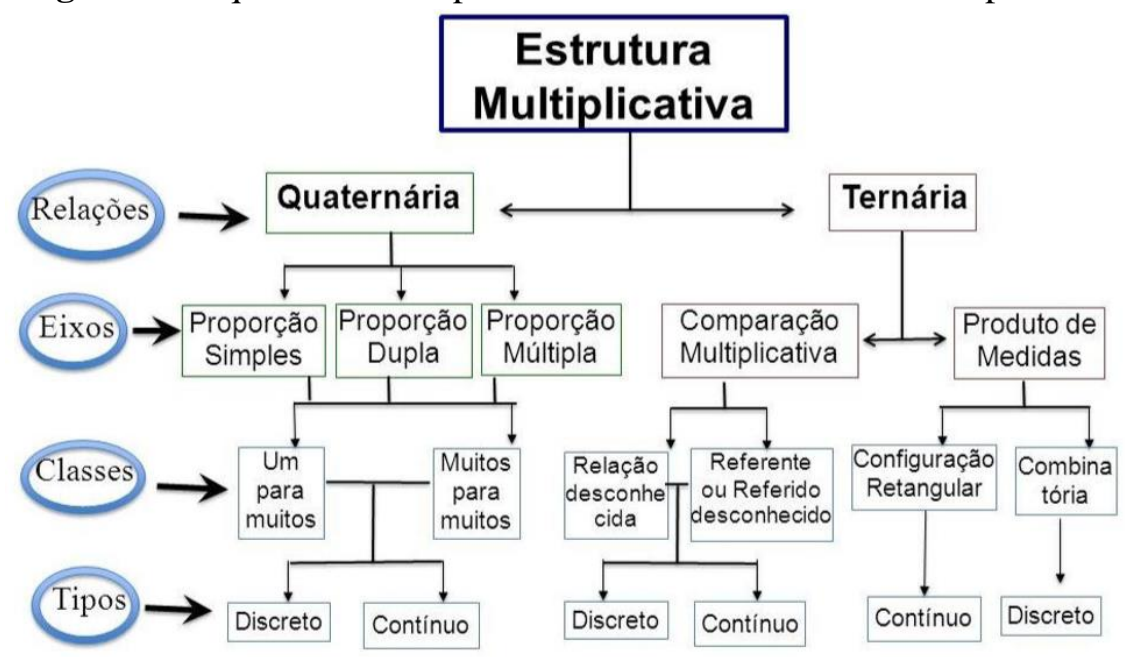

Fonte: Magina, Santos e Merlini (2016, p. 77)

$\mathrm{O}$ esquema apresenta as duas principais relações que configuram o Campo Conceitual Multiplicativo, a Relação Ternária e a Relação Quaternária que são subdivididas em eixos, classes e tipos de grandezas.

As relações quaternárias, foco deste estudo e em específico o eixo das proporções simples, expressam uma dupla relação entre duas ou mais grandezas de naturezas distintas, que se relacionam duas a duas. As situações dessa relação podem ser modeladas como função linear, por estabelecer proporção entre duas grandezas, como visto no exemplo 1 .

Exemplo 1: Um pacote de biscoito custa $\mathrm{R} \$ 3,00$. Quanto terei que pagar, caso compre três pacotes?

Figura 2 - Esquema de representação do exemplo 1

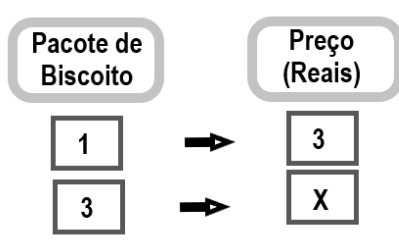

Fonte: os autores, 2021

Nesse problema, há uma relação entre as grandezas pacote de biscoito e preço. Ao realizar a multiplicação entre a quantidade de pacotes de biscoito por seu preço (em reais) o resultado será dado em reais, isto é, o valor encontrado será uma grandeza já conhecida, apresentando, portanto, uma estrutura de proporção entre duas grandezas. 
Os problemas especificamente do eixo Proporção Simples são caracterizados por apresentar uma relação entre quatro quantidades, tomadas duas a duas, de forma que duas são de uma grandeza e as outras duas são de outra grandeza. Desta forma, este tipo de situação forma uma simples proporção direta entre duas variáveis, como por exemplo entre tempo e distância ou entre pessoas e objetos. Gitirana et al (2014, p. 53) explicitam que problemas desta natureza "trazem situações em que se tem uma relação de proporcionalidade entre quatro grandezas - duas a duas de mesma espécie - que estão relacionadas por uma taxa entre as grandezas de diferentes espécies”. Essas situações são agrupadas em duas classes: a correspondência um para muitos e a correspondência muitos para muitos.

Problemas de correspondência um para muitos dizem respeito a situações em que a relação entre as variáveis está explicitada, como no exemplo 2 abaixo (relação um para cinco).

Exemplo 2: Num pacote há cinco bombons. Quantas bombons teremos em quatro pacotes iguais a este?

Já os problemas de correspondência muitos para muitos vão se referir às situações em que não se é possível conseguir a relação um para muitos ou a relação um para muitos é implícita à situação, como vemos nos exemplos 3 e 4.

Exemplo 3: A Mercearia Boas Compras está com uma promoção. Na compra de cinco pacotes de macarrão você ganha de brinde 3 pacotes de queijo ralado. Se eu comprar 15 pacotes de macarrão quantos pacotes de queijo ralado eu ganharei?

Exemplo 4: oito carros, juntos, têm 32 rodas. Quantas rodas temos em três carros juntos? Observa-se que, nesse caso, a relação um para muitos está implícita, porque podemos descobrir que um carro tem quatro rodas e, a partir daí, solucionar o problema.

Os problemas de Proporções Simples, sejam da classe um para muitos ou da classe muitos para muitos, podem ser solucionados por meio da utilização das operações de multiplicação e divisão. Sobre os problemas de divisão Vergnaud (1983; 1988; 2009), chama atenção para o fato de duas situações (divisão por partição ou por quotas) requerem a mesma operação matemática, mas apresentarem significados distintos. A situação de divisão por partição requer a distribuição de uma quantidade em partes, devendo-se encontrar o tamanho da parte (divisão de um todo em partes iguais), enquanto que a 
divisão por quotas requer a distribuição de uma determinada quantidade em quotas préestabelecidas (o todo é dividido pelo tamanho das partes, as quotas estabelecidas).

A diferença entre contar e medir leva às diferenças entre os tipos de grandezas (discretas e grandezas) presentes nas diversas situações do Campo Conceitual Multiplicativo, inclusive nas de Proporção Simples, uma vez que as grandezas contínuas diferem das discretas pelo seu caráter contínuo, como os comprimentos, as áreas, os volumes, a massa, a capacidade, etc. já que, quando medimos essas grandezas, sempre encontramos um valor intermediário, o que leva à necessidade dos números racionais.

Com as grandezas discretas é possível quantificar objetos e coisas em específico, como um livro ou uma pessoa, de forma que que não há uma quantidade intermediária entre as unidades (não temos, por exemplo, um terço de livro ou meia pessoa). Já com as grandezas contínuas a representação é feita através de uma unidade padrão que não pode ser separada do objeto, como oito metros de distância ou cinco quilos de feijão, porém, é possível a quantificação entre as unidades (podemos ter sete metros e 30 centímetros de distância ou cinco quilos e duzentos gramas de arroz).

Estudos recentes têm se dedicado a compreender a natureza dos problemas de Proporção Simples, na ótica dos Campos Conceituais, bem como quais as estratégias são utilizadas por estudantes na resolução de situações desse eixo e como estes estudantes pensam a respeito das situações apresentadas (LAUTERT; MERLINI; SANTOS; 2018; LAUTERT; SANTOS, 2018; SANTOS; SILVA; SILVA, 2019; MAGINA; LAUTERT; SANTOS, 2020). Nesta direção, é fu nd a ment al compreender a natureza dos diferentes tipos de situações que permeiam a sala de aula de matemática, pois tal compreensão pode auxiliar o ensino e a aprendizagem através de situações que propiciem uma compreensão conceitual ampla dos conteúdos matemáticos, neste caso o conhecimento sobre os conceitos que envolvem a proporcionalidade, e não apenas algorítmica.

\section{Metodologia}

O presente trabalho de pesquisa foi desenvolvido dentro de uma abordagem qualitativa, de cunho exploratório-explicativo, buscando uma interpretação aprofundada dos fatos, ou seja, a natureza dos problemas de estruturas multiplicativas, em específico as situações do eixo proporção simples, incluindo-se as classes, obtidas da análise de cada uma das provas objeto de estudo desta pesquisa. Este tipo de 
abordagem leva em consideração os vários componentes envolvidos em uma situação, a saber, como as provas de matemática e suas tecnologias do ENEM, pautadas nos programas oficiais da Educação Básica, abordam um conceito do campo multiplicativo (Proporção Simples) e consideram suas interações.

O procedimento de análise foi iniciado com a seleção dos itens, envolvendo o Campo Conceitual das Estruturas Multiplicativas, presentes em cada uma das provas do triênio proposto (2017-2019). Com os itens que abordavam explicitamente os conceitos deste campo, posteriormente, os itens foram selecionados considerando a relação (quaternária ou ternária) e apenas os problemas de relação quaternária passaram a ser analisados com relação ao significado e o conceito multiplicativo presentes na abordagem (proporção simples: multiplicação um para muitos; proporção simples: multiplicação muitos para muitos; divisão por partição; divisão quota; proporcionalidade de uma forma geral). Todos os itens selecionados foram resolvidos pelos pesquisadores, buscando identificar os invariantes operatórios e as possibilidades de representações simbólicas ${ }^{4}$ do conceito envolvido.

Todas as provas foram analisadas de forma independente pelos pesquisadores do estudo aqui descrito. As discordâncias foram analisadas por um terceiro avaliador, convidado a contribuir voluntariamente com o estudo, também de forma independente, cuja classificação foi considerada a final (a partir das análises inicialmente a ele apresentadas). Cabe ressaltar que a análise não contemplou itens das provas que envolviam apenas a computação numérica (algoritmo), tendo como base as justificativas para a construção do significado do conceito apresentado por Verganaud em sua teoria.

\section{Resultados e Discussões}

Em relação à análise das situações do Campo Conceitual Multiplicativo presentes nas provas de matemáticas e suas tecnologias do ENEM no último triênio (2017-2019),

\footnotetext{
${ }^{4}$ Vergnaud $(1988 ; 1994)$ indica que para o desenvolvimento e construção de determinado conceito se faz necessário o domínio de uma tríade de aspectos formada pela situação (dão sentido ao conceito), invariantes operatórios (objetivos, propriedades e relações sobre os quais repousa a operacionalidade do conceito) e as representações simbólicas (linguagem natural, gráficos, diagramas, dentre outros, que podem ser usados para indicar e representar os invariantes e, consequentemente, representar as situações e procedimentos).
} 
nota-se pelo quadro 1 que não há uma grande variância da quantidade de questões que envolvem este campo conceitual.

Quadro 1 - Situações multiplicativas por ano de aplicação do ENEM

\begin{tabular}{|c|c|c|}
\hline Ano do exame & $\begin{array}{c}\text { Quantidade de questões } \\
\text { da prova de matemática } \\
\text { e suas tecnologias }\end{array}$ & $\begin{array}{c}\text { Quantidade de questões } \\
\text { que abarcam o campo } \\
\text { conceitual multiplicativo }\end{array}$ \\
\hline 2017 & 45 & $\begin{array}{c}35 \\
(77,8 \%)\end{array}$ \\
\hline $2018^{*}$ & 44 & $\begin{array}{c}37 \\
(84,09 \%)\end{array}$ \\
\hline 2019 & 45 & $\begin{array}{c}37 \\
(82,2 \%)\end{array}$ \\
& & 109 \\
$(81,3 \%)$
\end{tabular}

Fonte: dados da pesquisa 2020.

Observa-se que o número de itens que aborda conceitos multiplicativos em cada prova, em cada ano, é considerado muito satisfatório, tendo em vista que a constituição das provas contempla $109(81,3 \%)$ das 134 questões propostas no triênio. Nesta direção, o ENEM tem valorizado o pensamento de Vergnaud $(1983 ; 1988 ; 1991)$ que afirma que será requerido do estudante um longo período de tempo para a sua apropriação conceitual, tendo em vista a grande diversidade de conceitos que envolve o Campo Conceitual Multiplicativo. Assim, o exame explora de forma ampla os conceitos, em vários itens, dentre os vários blocos temáticos dos conteúdos matemáticos, visando à apropriação dos conceitos deste campo conceitual, levando em conta o currículo da Educação Básica que é constituído, nesta direção, por todo Ensino Fundamental e Médio.

Com relação ao total de questões do Campo Multiplicativo identificadas e analisadas (109), apenas 38 questões $(34,86 \%)$ explicitamente puderam ser classificadas de acordo com o quadro referência de Magina, Santos e Merlini (2016). Isto porque este campo conceitual envolve vários conceitos, para além daqueles presentes nas situações destacadas no quadro referência da análise. Nesta direção, Vergnaud (1988) propôs uma classificação dos problemas considerando tanto as estruturas matemáticas associadas, quanto às dificuldades das situações-problema e os raciocínios necessários para resolvêlas.

Assim, os 71 itens $(65,14 \%)$ não classificadas neste estudo, e que permeiam as estruturas multiplicativas, tratam de conceitos e conteúdos matemáticos (frações, 
números racionais, porcentagem, taxa, função linear, função n-linear, análise dimensional, vetores, dentre outros) que abordam para além das relações quaternárias e ternárias, uma vez que estas relações apontam explicitamente para os conceitos de multiplicação e divisão (ou a combinação de ambos) na abordagem de situações de proporção simples, proporção múltipla, combinatória, etc. Destacamos, como já explicitado, que todos estes conceitos tratam dos esquemas de ação de distribuição, de equivalência e da correspondência um para muitos (base do Campo Conceitual Multiplicativo) como indicam os pressupostos de Vergnaud (1983; 1988; 2009).

Dos 38 itens classificados no estudo, 26 (68,42\%), conforme o quadro 2, são situações de relação quaternária e todos eles (100\%) abordam a Proporção Simples enquanto eixo.

Quadro 2 - Situações de proporção simples por ano de aplicação do ENEM

\begin{tabular}{|c|c|c|c|}
\hline Ano do Exame & $\begin{array}{c}\text { Questões classificadas de } \\
\text { acordo ao quadro } \\
\text { referência }\end{array}$ & $\begin{array}{c}\text { Questões de relação } \\
\text { quaternária }\end{array}$ & $\begin{array}{c}\text { Questões de } \\
\text { proporção simples }\end{array}$ \\
\hline 2017 & 9 & $\begin{array}{c}7 \\
(77,78 \%)\end{array}$ & $\begin{array}{c}7 \\
(100 \%)\end{array}$ \\
\hline 2018 & 12 & $\begin{array}{c}9 \\
(75 \%)\end{array}$ & $(100 \%)$ \\
\hline 2019 & 15 & 10 & 10 \\
& & $(66,67 \%)$ & 26 \\
\hline
\end{tabular}

Fonte: dados da pesquisa 2020.

Nessa ótica, Vergnaud (2009) aponta que a primeira grande forma da relação multiplicativa é compreendermos como se estabelece uma relação quaternária de Proporção Simples, na interligação de quatro quantidades, nas quais duas quantidades são medidas de um tipo e as outras duas são medidas de outro tipo. Porém, não explorar situações mais sofisticadas neste eixo, como as proporções múltiplas e duplas, que começam a ser abordados nos anos finais do Ensino Fundamental, geralmente como situações intituladas como de "regra de três composta", muito presentes em livros didáticos, evidencia uma elaboração de situações em desequilíbrio do ponto de vista conceitual, no que tange a exploração das grandezas diretamente e inversamente 
proporcionais, que permeiam as competências e habilidades avaliados no ENEM (em todos os blocos temáticos dos conteúdos matemáticos).

Sobre as classes dos problemas de Proporção Simples presentes nos exames, 16 $(61,53 \%)$ são situações de um para muito e $10(38,47 \%)$ são situações de muitos para muitos como vemos no gráfico 1.

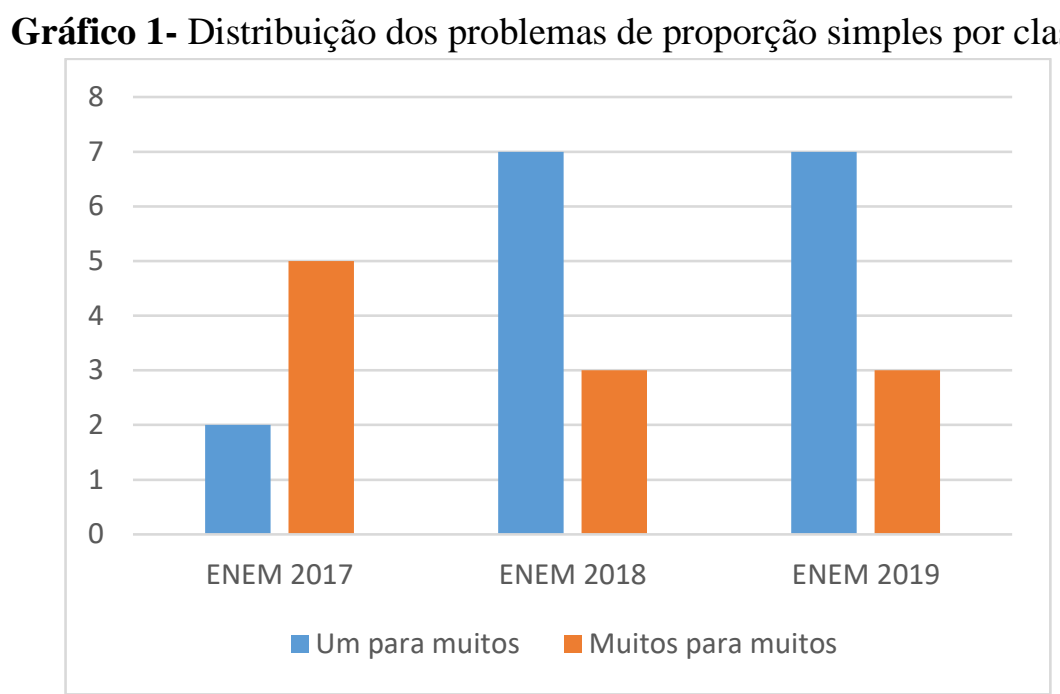

Fonte: dados da pesquisa 2020.

Evidencia-se que nas provas do ENEM, no que se refere aos problemas de Proporção Simples, estão mais presentes situações pouco sofisticadas com relação aos invariantes operatórios e as representações simbólicas (problemas da classe um para muitos). Acreditamos que isso se deva em função da relação tempo de realização da prova versus volume de questões a serem respondidas, o que implica em questões menos complexas do ponto de vista conceitual, mas que abordam explicitamente as habilidades e competências que são foco da avaliação do ENEM. Assim, por exemplo, é possível que os estudantes resolvam estes itens apenas com o emprego direto da multiplicação ou da divisão, operações elementares, podendo utilizar inclusive raciocínio aditivo para este tipo de problema, corroborando com os resultados de Lautert, Merlini e Santos (2018) e Santos e Lautert (2018).

Nesta direção, a sofisticação do ponto de vista cognitivo, das situações-problema presentes no ENEM, está no fato de requererem, muitas vezes, o emprego do raciocínio proporcional das classes um para muitos e muitos para muitos num mesmo item, como no caso do problema apresentado na figura 3 , ou no emprego do raciocínio de muitos para 
muitos em mais de um esquema de representação simbólica no mesmo item, como acontece no problema da figura 4 , como exemplos.

Figura 3 - Situação problema que envolve as classes um para muitos e muitos para muitos

\section{QUESTÃO 150}

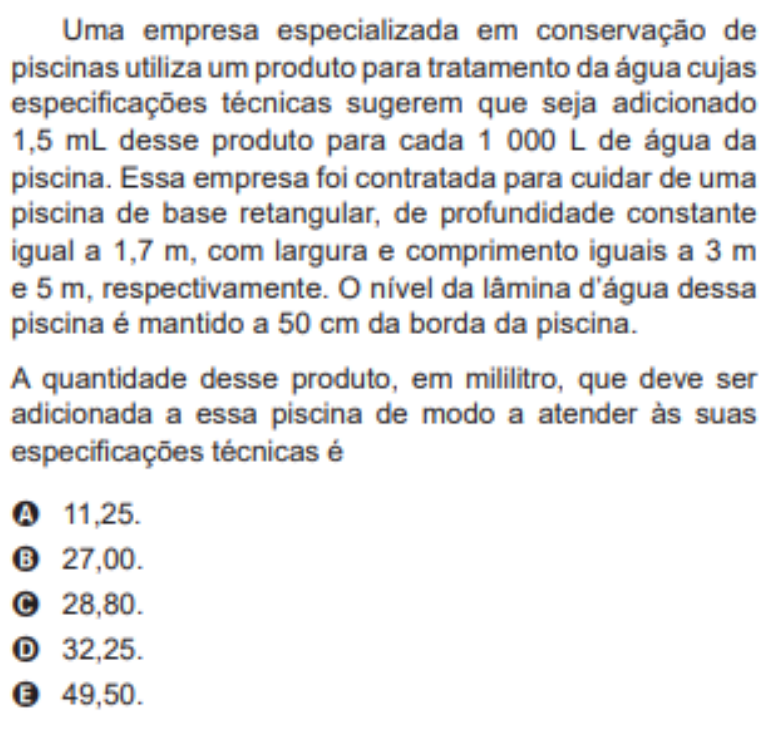

Fonte: INEP (2017)

Para resolução da questão 150 da prova do ENEM de 2017, é necessário empregar o raciocínio proporcional da classe um para muitos para descobrir que $18 \mathrm{~m}^{3}$ correspondem a 18.000 litros (se $1 \mathrm{~m}^{3}$ equivale a 1.000 litros) e, em seguida, aplicar o esquema de resolução de uma proporção simples relacionando mililitros com litros (neste caso, aplica-se o raciocínio proporcional de muitos para muitos). 
Figura 4 - Situação-problema que envolve a classe muitos para muitos em mais de um esquema

\begin{tabular}{l} 
QUESTÃo 152 \\
Os tipos de prata normalmente vendidos são 975, \\
950 e 925 . Essa classificação é feita de acordo com a \\
sua pureza. Por exemplo, a prata 975 é a substância \\
constituida de 975 partes de prata pura e 25 partes de \\
cobre em 1000 partes da substância. Já a prata 950 é \\
constituída de 950 partes de prata pura e 50 de cobre em \\
1 000; e a prata 925 é constituida de 925 partes de prata \\
pura e 75 partes de cobre em 1 000. Um ourives possui \\
10 gramas de prata 925 e deseja obter 40 gramas de \\
prata 950 para produção de uma joia. \\
\hline Nessas condiçōes, quantos gramas de prata e de cobre, \\
respectivamente, devem ser fundidos com os 10 gramas \\
de praț 925 ? \\
Q 29,25 e 0,75 \\
B 28,75 e 1,25 \\
C) 28,50 e 1,50 \\
(D) 27,75 e 2,25 \\
E 25,00 e 5,00
\end{tabular}

Fonte: INEP (2018)

Na resolução da questão 152 da prova do ENEM 2018, encontramos o valor de 10 gramas a partir da relação proporcional entre 975 partes de prata e 1000 gramas e, em seguida, aplicamos a relação proporcional entre 950 partes de prata e 1000 gramas para as partes de prata correspondentes a 40 gramas.

\section{Considerações finais}

Com relação aos objetivos do presente estudo, inferimos que o Campo Conceitual Multiplicativo foi bem explorado nas Provas do ENEM no triênio 2017-2019, sendo a maioria das questões relacionadas a conceitos não atrelados às relações quaternárias e ternárias, o que evidencia uma sofisticação conceitual das situações-problemas propostas, abordando de forma ampla os conceitos multiplicativos. Isto é esperado no Ensino Médio, final da etapa da Educação Básica, principalmente no que tange o currículo escolar.

Especificamente com relação aos itens que exploram as Proporções Simples, o exame precisa abordar de forma mais abrangente situações que envolvam grandezas diretamente e inversamente proporcionais, tendo em vista a pouca sofisticação conceitual na exploração dos invariantes operatórios e as representações simbólicas na resolução dos itens desse eixo, uma vez que a ênfase é dada a problemas da classe um para muitos.

Apesar de amplamente empregada na Educação Básica e ser referência na Prova de Matemática e suas Tecnologias no ENEM, a metodologia da resolução de problemas 
ainda precisa ser mais explorada no processo de ensino e aprendizagem sob a ótica dos conceitos envolvidos nas situações abordadas, independente das habilidades e competência requeridas nos problemas explorados. Apesar de reconhecerem sua importância, muitos professores e futuros professores não conhecem todos os aspectos dessa metodologia quanto a formulam e propões situações para resolução dos estudantes no trabalho com a matemática escolar.

\section{Referências}

BARRETO, A. L. de O.; RÊGO, R. G. Multiplicative structures in the form(action) of teachers from elementary school in a school of Fortaleza. Educação \& Formação, 5 (3), p. 1-19, 2020.

D’AMBRÓSIO, U. Educação Matemática: da teoria à prática. Campinas: Prós, 1996.

DANTE, L. R. Formulação e resolução de problemas de matemática: teoria e prática. São Paulo: Ática, 2009.

DEMO, P. Desafios modernos da Educação. Petrópolis: Vozes, 2000.

GITIRANA, V., MAGINA, S., CAMPOS, T.; SPINILLO, A. Repensando

Multiplicação e Divisão: contribuições da Teoria dos campos Conceituais. São Paulo: Editora PROEM, 2014.

LAUTERT, S. L.; MERLINI, V. L.; SANTOS, E. M. Resolução de Problemas de Divisão de Proporção Simples por Estudantes do 3 e 5 Anos. Anais do VII Seminário Internacional de Pesquisa em Educação Matemática. Foz do Iguaçu, p. 1-13, 2018.

LAUTERT, S. L.; SANTOS. Estudantes do 10 ano ao 3o ano resolvem situações multiplicativas. In: Síntria Labres Lautert; José Aires de Castro Filho; Eurivalda Ribeiro dos Santos Santana. (Org.). Ensinando multiplicação e divisão do 10 ao 30 ano. Série Alfabetização Matemática, Estatística e Científica. Coletânea Cadernos E-Mult. 1ed.Itabuna -Bahia: Via Litterarum, v. 1, p. 45-76, 2017.

MAGINA, S. M. P.; LAUTERT, S. L.; SANTOS, E. M. Estratégias Exitosas de Alunos dos Anos Iniciais em Situações de Proporção. Educação e Realidade, v. 45, p. 1-24, 2020.

MAGINA, S., MERLINI, V. L.; SANTOS, A. A Estrutura Multiplicativa à luz da Teoria dos Campos Conceituais. In: J. A. Castro Filho (Ed..). Matemática, cultura e tecnologia: perspectivas internacionais. Curitiba: CRV, p. 65-82, 2016.

POLYA, G. A arte de resolver problemas. Rio de Janeiro: Interciências, 1995. 
SANTANA, E. R. S.; LAUTERT, S. L.; CASTRO-FILHO, J. A.; SANTOS, E. M.. Observatório da Educação em Rede: As Estruturas Multiplicativas e a Formação Continuada. Educação Matemática em Foco (UEPB), v. 5, p.77-96, 2016. SANTOS, E. M., SILVA, M. C. N.; SILVA, S. C. A abordagem de proporção simples em um livro didático de matemática na ótica do campo conceitual multiplicativo. Revista Aquila. 21(9), p. 153-165, 2019.

SANTOS, E. M.; LAUTERT, S. L. A resolução de situações de proporção simples por estudantes dos anos iniciais do ensino fundamental. Anais do 5 Simpósio

Internacional de Pesquisa em Educação Matemática, V.01, Belém, p. 1-14, 2018.

SPINILlO, A. G., LAUTERT, S. L., BORBA, R. E., SANTOS, E. M.; SILVA, J. F. G. Formulação de problemas matemáticos de estrutura multiplicativa por professores do ensino fundamental. Bolema. 59(31), p. 928-946, 2017.

VERGNAUD, G. A gênese dos campos conceituais. In: E. Grossi (Ed.), Por que ainda há quem não aprende? A teoria. Petrópolis: Editora Vozes, p. 21-60, 2003.

VERGNAUD, G. Multiplicative structures. In: R. Lesh \& M. Landau (Eds.), Acquisitions of mathematics concepts and procedures. New York: Academic Press, p. 127-174, 1983.

VERGNAUD, G. Multiplicative structures. In: H. Hiebert \& M. BEHR, M. (Eds.), Research Agenda in Mathematics Education. Number Concepts and Operations in the Middle Grades. Hillsdale, N.J.: Lawrence Erlbaum, p. 141-161, 1988.

VERGNAUD, G. El niño, las matemáticas y la realidad: problemas de la enseñanza de lãs matemáticas em la escuela primária. México: Trillas, 1991.

VERGNAUD, G. Teoria dos campos conceituais. In: L. Nasser (Ed.), Anais do $\mathbf{1}^{\mathbf{0}}$ Seminário Internacional de Educação Matemática do Rio de Janeiro. P. 1-26, 1993.

VERGNAUD. Multiplicative conceptual field: what and why? In. H. Gershon \& J. Confrey. (Eds.), The development of multiplicative reasoning in the learning of mathematics. Albany, N.Y.: State University of New York Press, p. 41-59, 1994.

VERGNAUD, G. A Teoria dos Campos Conceituais. In: J. Brun. (Ed.), Didáctica das Matemáticas. Lisboa: Instituto Piaget, p. 155-191, 1996.

VERGNAUD, G. A criança, a matemática e a realidade. Curitiba: Editora da UFPR, 2009. 